\title{
Fluid Circuit Technology: Integrated Interconnect Technology for Miniature Fluidic Devices
}

\author{
Don VerLee, A. Alcock, G. Clark, T. M. Huang, S. Kantor, \\ T. Nemcek, J. Norlie, J. Pan, F. Walsworth, S. T. Wong.
}

\author{
Abbott Laboratories Hospital Products Division \\ Abbott Park, Illinois, 60064-3537
}

\begin{abstract}
According to many in the MEMS field, medical markets currently represent the second biggest opportunity for microfabricated devices and systems [1]. Certainly, microfabricated blood pressure sensors have already found a foothold in the industry. However, packaging and interconnections between devices remain major barriers to more wide-spread use of MEMS in medical applications.

It has become evident to us that the application of MEMS to medical diagnostics is hindered by the lack of an intermediate scale fluid handling system for feeding microscopic elements. In this paper, we describe a technology that may be able to serve as an interconnect technology between microfabricated devices and as a transition between macroscopic and microscopic scales. We describe a manufacturing process, miniature fluidic elements, and system designs that may offer useful insights into how one might design a microfabricated fluid circuit system.
\end{abstract}

\section{INTRODUCTION}

The future direction for many medical diagnostic analyzers portends increasing fluid processing complexity, miniaturization, and reliability while the costs to manufacture and to operate decrease. The factors that are driving these changes include; cost containment pressures, a steady increase in the menu of tests offered and the resulting number of accessible diagnostic reagents, the need to automate front end sample processing, the need to reduce the fluid volumes of reagent and sample used, and the desire to reduce the amount of solid waste generated as a diagnostic test is run.

Diagnostic instrument and medical device designers have watched developments in MEMS technology for years hoping to apply it to solve this growing list of challenges, yet they still see barriers that limit the spectrum of its applicability. For in-vivo and in-vitro diagnostic use, protein adsorption and clotting of small passageways top the list of concerns about biologically compatible materials and surface treatments. In addition there is a fundamental need to provide an interface between the macroscopic world, where samples are aspirated and dispensed in milliliter quantities, and the microscopic device which has internal volumes of nanoliters to microliters.

It is unrealistic to assume that the macroscopic fluid volumes needed in current instrumentation can all be driven down to microscopic volumes in the future. The lower limit of concentration for some bacterial infections can be as small as one cell per milliliter of blood. It should be clear that there is a lower limit on the volume of sample that must be evaluated to assure statistically relevant results even with multiplication factors that exceed $10^{15}$.

To address many of these pressing needs for handling fluids in future clinical diagnostic analyzers and medical devices, Abbott Laboratories has advanced the development of a technology that miniaturizes and integrates fluidic structures and control elements into solid blocks of acrylic - Fluid Circuit Technology.

The objective of the Fluid Circuit Technology program was to investigate and develop fundamental technology to enable the miniaturization and integration of the fluid handling functions needed in a typical medical diagnostic analyzer. The program encompassed research on miniature valves, chambers, channels, fluid metering, and mixing structures and methods, and a manufacturing process to combine these elements into a single integrated network. An additional goal was to demonstrate that this technology is functionally equivalent to existing diagnostic instrumentation while still providing the added benefits of reduced reagent and sample consumption, reduced solid waste, reduced size and cost of the instrument, and increased overall reliability.

The four aspects of Fluid Circuit Technology that will be described below are; A Process for Manufacturing Fluid Circuits, Fundamental Fluid Circuit Elements, Fluid Processing Subsystems, and examples of Fluid Circuit Systems. From the examples, parallels with MEMS technology in wafer bonding, valve design, and system design should become evident.

\section{FLUID CIRCUIT MANUFACTURING PROCESS}

Many techniques exist for bonding thermoplastics. Some of the more common techniques include solvent cementing, ultrasonic welding, adhesives, and thermal welding [2]. At the core of Fluid Circuit Technology is a process for bonding layers of thermoplastic into which fluidic structures such as channels and chambers have been machined [3]. This bonding process has the advantage over other techniques in its ability to create solid seamless blocks of plastic, without flash or bubbles, while retaining the internal three-dimensional fluid circuit detail. Originally developed and patented by companies such as Eastern Plastics, Plainville, Conn. and Carville, Dorking, England, we have investigated and optimized this process further for use in medical diagnostic instrumentation.

Fluid circuits have been fabricated out of a variety of thermoplastics, including some injection molded plastics, but the majority of the fluid circuits have been made out of cast polymethyl-methacrylate using the 5 step process that follows.

\section{Preparing Fluid Circuit Raw Materials:}

The initial process, called "shrinking", is needed to minimize dimensional drift in the bonding process, that may be caused by unrelieved stresses in the starting material. It involves baking unconfined sheets of the plastic that have been cut down to approximate size, at an elevated temperature $\left(152^{\circ} \mathrm{C}\right.$ for cast acrylic) for 10 hours, followed by a gradual cool down to room temperature. This usually causes these "blanks" to expand in thickness, and shrink in both of their other axes. The result is a block of plastic that is relieved of internal stresses, and that is relatively dimensionally stable over subsequent heating cycles. 


\section{Machining the Fluid Circuit Layers:}

To begin the process of machining the channels and chambers that make up a fluid circuit, the blanks that have been "shrunk" are squared on a milling machine, annealed in an oven ( $90^{\circ} \mathrm{C}$ for $1 \mathrm{hr}$. for cast acrylic), then fixtured in a computer numerical controlled milling machine. The fluid circuit design itself is downloaded into the $\mathrm{CNC}$ as a list of tools and an extensive sequence of tool path coordinates in much the same manner that apertures and Gerber photoplot vectors are downloaded into a photoplotter to define the layers of a printed circuit board.

To create cylindrical channels and chambers, mirror images of the tool paths are replicated on each opposing face of the acrylic blocks that are to be bonded. The coordinates for the machining of these features are referenced from two alignment holes that are machined into the faces at opposing corners of each block. Dowel pins placed in these holes insure that when the faces are brought together both the top and bottom halves of the cylindrical cavities line up.

Several factors determine how extensive the machining process becomes. The largest contributor to machining time is the surface finish required on the inside of the chambers. In medical diagnostic instrumentation, significant attention is paid to the chemical interactions at the interfaces between solids and liquids to minimize problems such as proteins adsorbing to surfaces, carryover, uneven wetting, bubble entrapment, fluid metering imprecision, and uncontrolled side reactions. We have explored several approaches to minimizing machining time while optimizing surface finish including; flame and vapor polishing, mechanical polishing, and diamond tooling. Of these, the best surface finishes can be achieved in the least amount of time with diamond tooling.

Care is taken during both the layout and machining of the fluid circuit to assure that no channels or holes penetrate to what will eventually become the outside of the bonded block of acrylic. This is done to prevent uneven heating and collapsing of these channels during the bonding process.

A final machining step is to fly-cut the faces to be bonded with a diamond fly cutting tool. The quality of the bond is dependent on the surface finish and flatness of the two faces to be bonded and unless the edges and side walls of the channels and chambers are sharp, a seam defect will be evident after the bonding is complete.

\section{Thermal Diffusion Bonding Fluid Circuit Layers:}

After machining, the fluid circuit layers are annealed, washed with detergent and water, and inspected before they are bonded. The flat polished faces of the blocks to be bonded are aligned with dowel pins and stacked in the order that they are to be bonded inside a bonding fixture. A cross section through a bonding fixture is illustrated in Fig. 1 with the layers of a fluid circuit in it ready to be bonded. The bonding fixture is simply a steel base with a cavity in it and a floating lid. The fluid circuit layers to be bonded are machined to achieve a line to line fit when placed inside this cavity at room temperature. A cap piece is bolted onto the base with a series of springs that can be made to apply a known pressure onto the lid by compressing the springs a known distance.

Once the fluid circuit layers are stacked inside the fixture, the bolts are tightened down on the cap piece until a known pressure ( $\sim 45$ psi. for acrylic) is applied to the stack. The entire assembly is then placed in a programmable oven. A typical bonding cycle for cast acrylic includes a $2 \mathrm{hr}$. ramp to $126^{\circ} \mathrm{C}$,

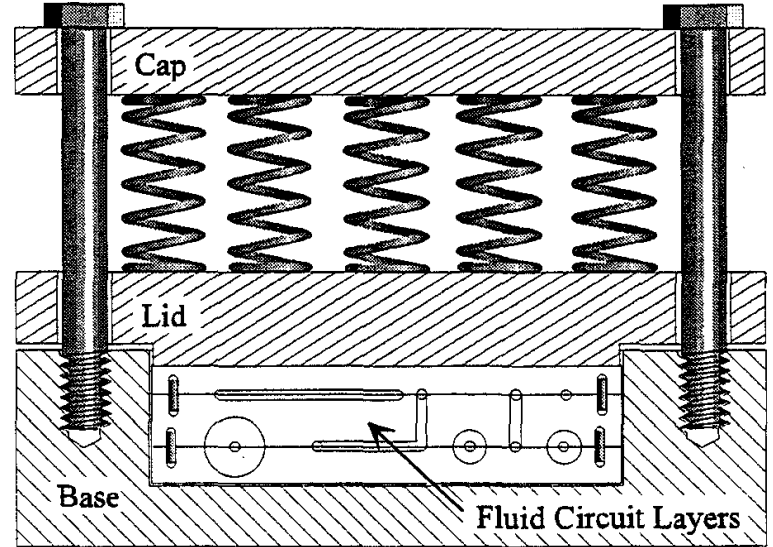

Fig. 1 Fluid Circuit Bonding Fixture

followed by a $4 \mathrm{hr}$. hold at that temperature, followed by a $2 \mathrm{hr}$. ramp down to room temperature.

The temperature at which bonding takes place is just above the glass transition temperature of the plastic. To assure that the internal features are maintained, this temperature must not be exceeded. The exact mechanism by which the bonding occurs is not fully understood, but at these elevated temperatures, the layers merge into one solid block, while retaining the fluid circuit detail that was machined in prior to bonding. The network of channels and chambers appear to "float" inside the block.

The best results we have obtained have been with cast polymers such as PolyCast Acrylic [4], polycarbonate, $\alpha$ butylstyrene, and others. In general, highly cross-linked and highly crystalline polymers don't bond well using this method, although regions (e.g. in channels) that have been surface crosslinked through plasma or chemical treatment, and crystalline polymer films have been successfully captured between bonded layers. Bonding injection or compression molded fluid circuit layers is possible but difficult to optimize, because it is difficult to eliminate undesirable characteristics introduced during molding processes; high variance in the glass ransition temperature, sink marks in thick cross sections, skinning, high unrelieved internal stresses, and rounded edges on the channel detail.

\section{Post-bond Machining:}

When the bond cycle is completed external features such as sensor pockets, external fluid connector ports, valve dimples, and mounting threads are added to the block. Initially any dimensional drift that may have occurred to internal features, during bonding is measured and used to adjust the coordinates of critical features in the original fluid circuit layout tool path code. Since this drift is fairly repeatable $(\sim \pm 25 \mu \mathrm{m})$ later "as built" fluid circuits match the design to within $\sim \pm 10 \mu \mathrm{m}$ near the center and within $\sim \pm 100$ $\mu \mathrm{m}$ near the edges of a large $20 \times 25 \mathrm{~cm}$ block.

The block is then aligned in the CNC machine so that the machine's coordinate system references the guide marks. All external features are then machined into the block with the same precision and accuracy as the internal detail of the fluid circuit. After this is complete and the block has been thoroughly washed and inspected the fluid circuit is ready for assembly.

\section{Assembling a Fluid Circuit:}

The assembly requirements differ for each fluid circuit and depend entirely on the complexity of the fluid circuit. For a simple fluid circuit the only assembly required, other than to mount fluid connectors to the block, is to create the valves. These 
simple valves are assembled in one step [6]. The process sandwiches a thin film $(12 \mu \mathrm{m})$ of pressure sensitive adhesive backed mylar (Flexcon V95 [5]) between two fluid circuit blocks. These two blocks will be called the "liquid side" and the "air side" (one for handling the biological liquids, the other for plumbing pneumatic control channels to each valve site). The air side fluid circuit is placed on a flat surface with the valve side facing up, and a sheet of the mylar film large enough to cover the block is simply rolled on starting at one edge of the block. Care must be taken not to introduce any wrinkles or dust particles under the film. The film is then carefully cut away from each bolt hole, dowel pin and fluid pass through location. Before the fluid side block is bolted on, a flat and highly polished backing plate is first bolted on and allowed to sit for 4 hrs. to make sure that the adhesive on the back of the film takes a set. The excess film is then trimmed away from the outside of the block and fluid connectors are mounted onto the assembled fluid circuit.

\section{FLUID CIRCUIT ELEMENTS}

If one were to draw the analogy that a fluid circuit was the fluidic equivalent of an electronic printed circuit, then a simple fluid circuit would consist of nothing more than channels and interconnection sites for externally mounted active components. However for a fluid circuit to be useful, structures which have no analogy to printed circuits are needed for fluid processing in addition to the channels. For example, it is possible to integrate fluid control elements such as valves and sensors, directly into the fluid circuit block itself and thus greatly reduce the "dead volume", sealing difficulties, and alignment problems encountered with mounting elements outside a fluid circuit.

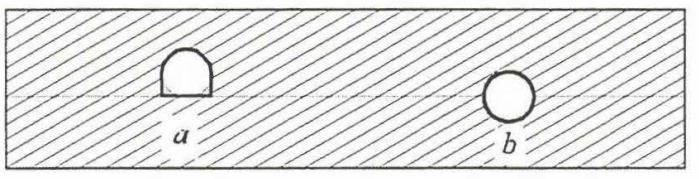

Fig. 2 "D" and Cylindrical Fluid Circuit Channels

The most fundamental fluid circuit element is a channel. The two basic fluid channel designs are illustrated in Fig. 2. Both of these are machined into the plastic using a ball end mill. The " $D$ " channel shape illustrated in Fig. 2a is the most routinely used fluid circuit geometry. " $D$ " channels are used extensively for plumbing pure fluids (reagents, diluents etc.), fluid sources, sinks, and air distribution networks. This channel shape is the least costly to machine since it can be fabricated in one pass on a single side of a block.

The cylindrical channel shape illustrated in Fig. $2 b$ is the preferred geometry for all liquid networks that directly handle biological liquids or the assay liquids. It can be clearly seen that the " $D$ " channel has two corners where liquid menisci remain when a channel is emptied. The fluid entrained in these areas causes higher carryover (or requires a greater volume of wash fluid to purge), which isn't a problem on cylindrical channels

The most common channel sizes used have been $0.8 \mathrm{~mm}, 1$ $\mathrm{mm}$ and $1.6 \mathrm{~mm}$ in diameter. Smaller channel diameters have been fabricated, $(0.5 \mathrm{~mm})$ but still smaller diameters have not been explored extensively since fluid flow resistance, probability of plugging and vapor lock all increase with decreasing diameter.

A variety of valves can be fabricated [6] inside a fluid circuit including those pictured in Figs. 3, and 4. Each of the fluid valves

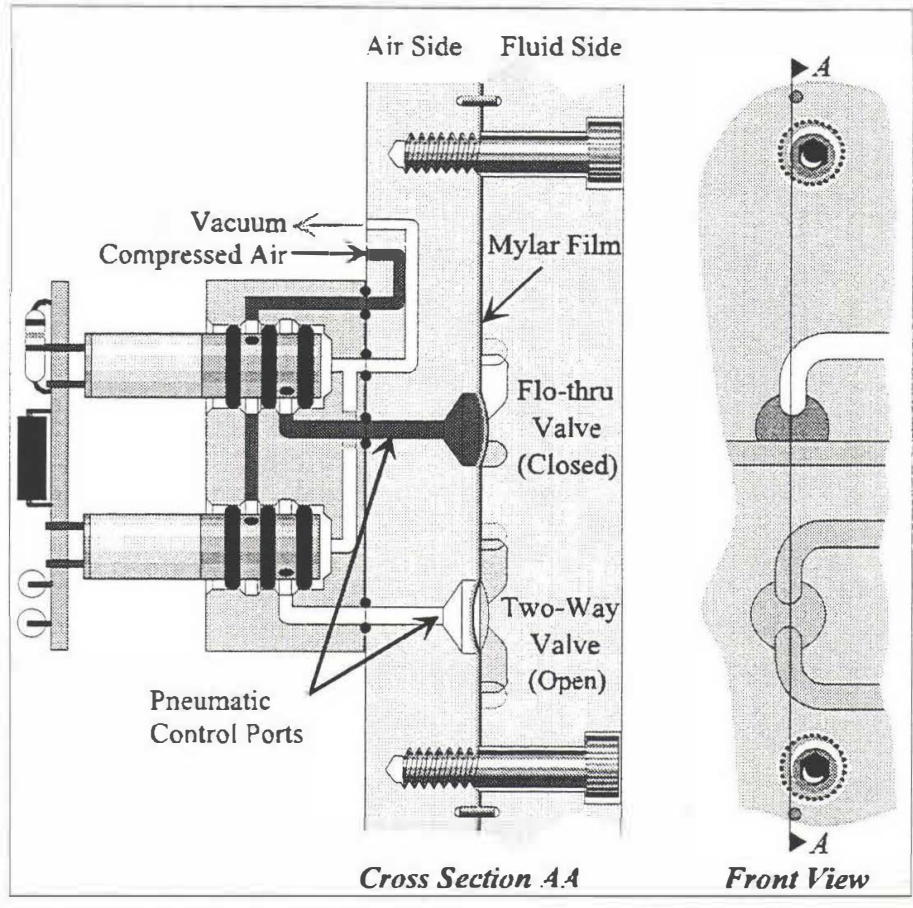

Fig. 3 Two-way and Flow-Through Fluid Circuit Valves

illustrated is pneumatically activated by a remote pilot valve which can be a miniature 3-way solenoid valve such as those from the Lee Company [7] pictured in Figs. 3 and 7. Remote pneumatic actuation results in a significant improvement in reliability of miniature fluid control systems. The difference in reliability between commercially available electromechanical liquid valves, and electromechanical pneumatic valves is from $10-100 \mathrm{X}$ number of total cycles before failure

The two general fluid circuit valve types illustrated in Fig 3. are 2-Way and Flow-Through valves. As can be seen in this schematic, 2-Way valves provide simple on/off control of fluid flow. Flow-Through valves, however, provide more interesting and useful control. They enable one fluid to merge into the middle of a free flowing channel at a fixed location and with very little "dead volume". This valve type enable many useful fluid circuit constructs including circuits that wash the channels out, and circuits that enable reagent metering, and mixing. A variant which has two flow through channels creates a cross-over point for fluid

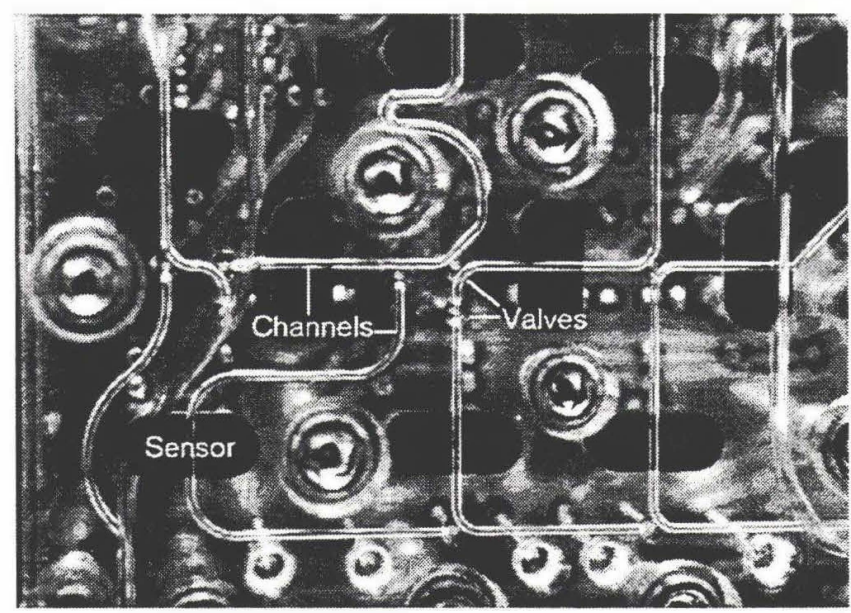

Fig. 4 Fluid Circuit Channels, Valves and Sensors 


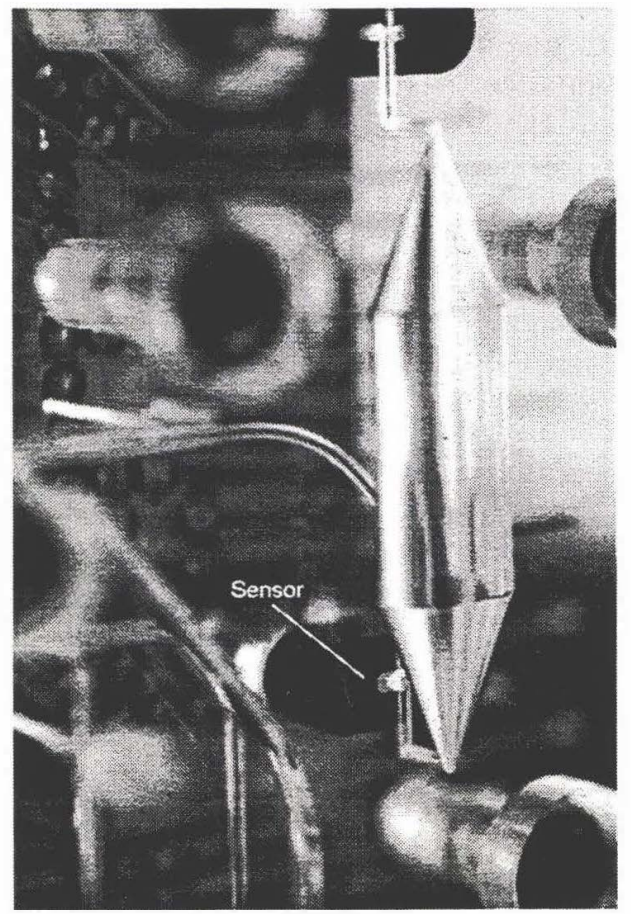

Fig. $510 \mathrm{ml}$ Metering Chamber \& Sensors

streams to cross from one flow network into another. Examples of these uses for Flow-Through valves can be seen in Fig. 4.

A variety of different chamber sizes and styles have also been fabricated inside a fluid circuit. Chambers used to meter larger volumes (Fig. 5), mix fluids, incubate mixtures (Fig. 6), provide reagent and diluent storage and provide waste sumps have been designed and tested. The incubation chambers shown in Fig. 6 have a cavity machined around them that has been filled with thermally conductive epoxy and assembled contacting a flexible heater attached to an aluminum plate. This created a "hot block" inside the fluid circuit to incubate assay reaction mixtures.

To provide feedback for controlling the fluid progress through a fluid circuit, sensors have been developed which can distinguish between air and liquid in the channel [8]. The sensors pictured in Figs. 4, 5, \& 6, are composed of an LED and a photodiode oriented in an opaque housing in such a manner so as to illuminate and reflect off a channel. A logic HI signal is generated if air is present, logic LO if liquid is present.

\section{FLUID CIRCUIT SUBSYSTEMS}

The elements that make up a fluid circuit have been combined to create functional subsystems. These have been utilized routinely either as repeated designs copied from one successful fluid circuit to another, or as stand-alone modules that connect to a larger fluid circuit system to carry out a function.

Several of these fluid circuit subsystems are illustrated in the figures. Fig. 7 illustrates a manifold of 16 three-way solenoid valves. This fluid circuit design provides compressed air and vacuum to each of 16 miniature Lee valves and enables a simple face-mount, leak free connection of each of the valves' common outputs to a fluid valve in a fluid circuit. The fluid circuit provides both compressed air and vacuum to the module and makes the connections from the module to each fluid valve control point. The drive electronics enable direct digital access to the module and can be modeled as a 16-bit memory word.

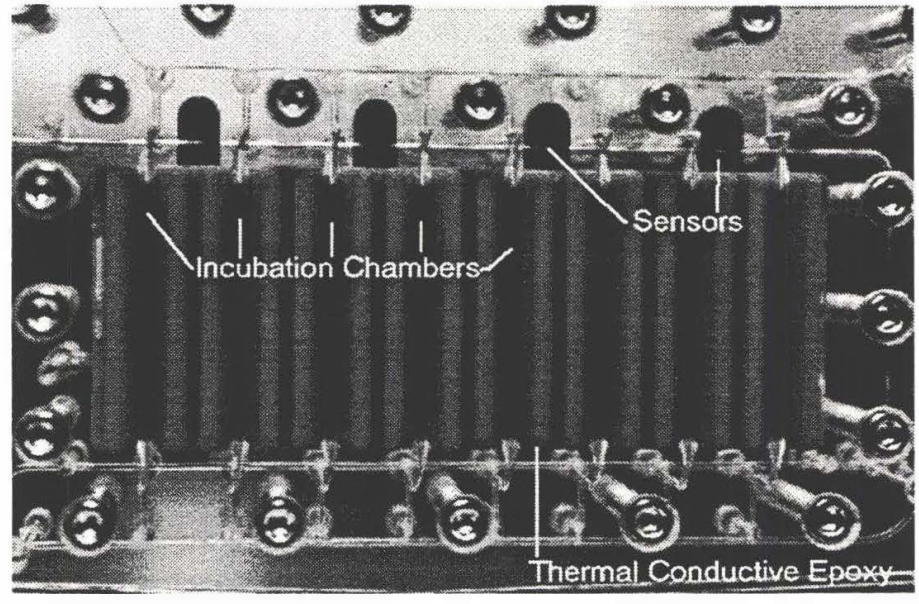

Fig. 6 Incubation Queue of 8 Chambers

Several fluid metering circuits are also pictured in Figs. 4, 5, and 8 . In just one example, Fig. 4 illustrates a close-up of a sensor to sensor fluid metering circuit that makes up the center section of the fluid circuit system pictured in Fig. 8. This circuit was designed to allow one fluid (sample or reagent) to be metered across a Flow-Through valve into another fluid by advancing the meniscus of the fluid to be metered from one sensor to another.

\section{FLUID CIRCUIT SYSTEMS}

Once initial feasibility had been demonstrated for fluid circuit elements, and subsystems, it became evident that Fluid Circuit Technology could provide a new fluid interconnect and control strategy for entire diagnostic instrument systems. The design approaches used in the development of complex electronic circuits could be applied to the design and development of a complex fluid control system. Proven functional modular subsystems based on simple circuit elements could be simply face or edge connected to other fluid circuits to make a reliable fluid circuit instrument system.

A feasibility project to demonstrate this concept was undertaken in 1992. The objective of the project was to demonstrate that a fluid circuit based instrument could produce comparable performance (throughput, assay sensitivity and specificity) to an existing commercial instrument. Implementing this hierarchical design philosophy meant that the design team had to abandon traditional instrument design approaches that utilized robotics and disposable plastic containers to meter, mix and manipulate fluids, and focus instead on what needed to be done with the fluids to carry out a typical diagnostic assay chemical reaction.

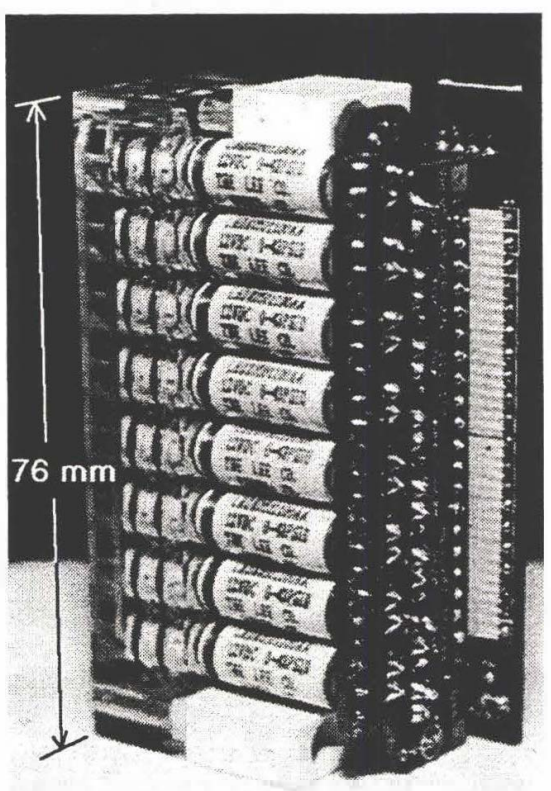

Fig. 7 Miniature 16 Valve Manifold 


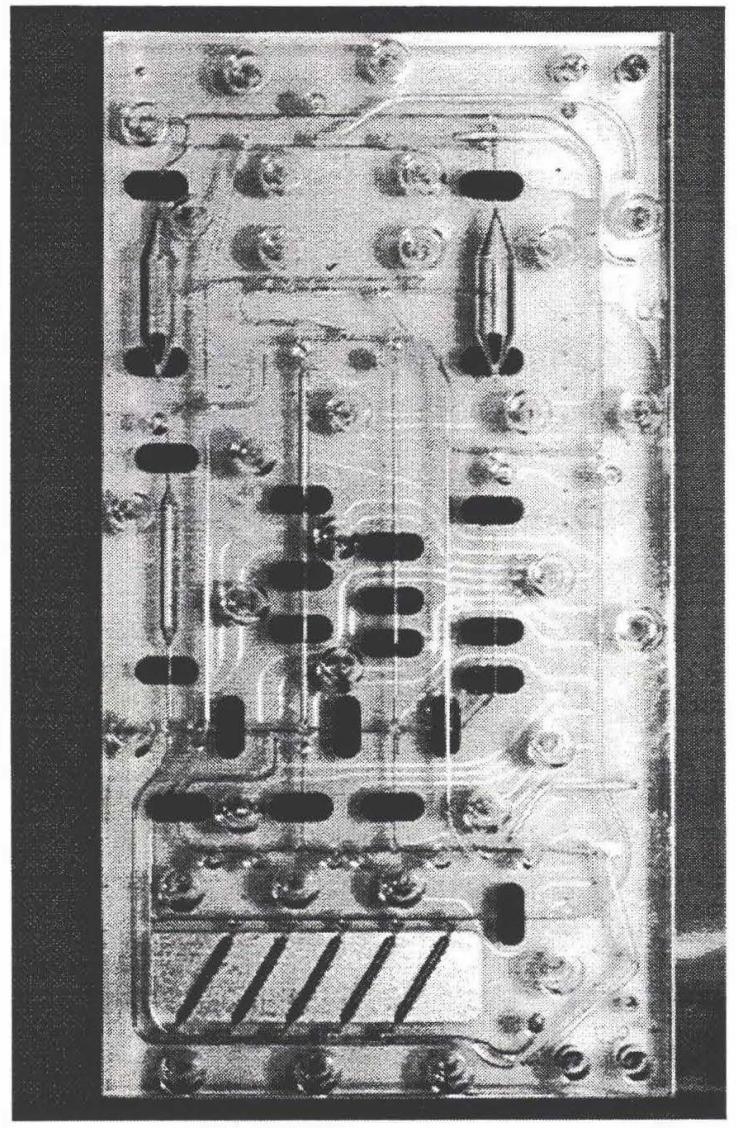

Fig. 8 Miniature Low Volume Clinical Chemistry Fluid Circuit System

The first instrument system chosen for comparison was Abbott's $\mathrm{TD}_{\mathrm{x}}^{\mathrm{TM}}$ instrument. The $\mathrm{TD}_{\mathrm{x}}^{\mathrm{TM}}$ assay system includes a sensitive and completely automated diagnostic instrument, and more than 60 different assays for measuring the concentration of various therapeutic drugs. Each assay's sensitivity is specified in the $\mathrm{ng} / \mathrm{ml}$ range, and their specificity has been determined against common known interferents. The instrument is a batch analyzer with a carousel capable of handling 20 patient samples at a time with a throughput of $60-80$ tests per hour.

To develop a fluid circuit equivalent of this analyzer, the

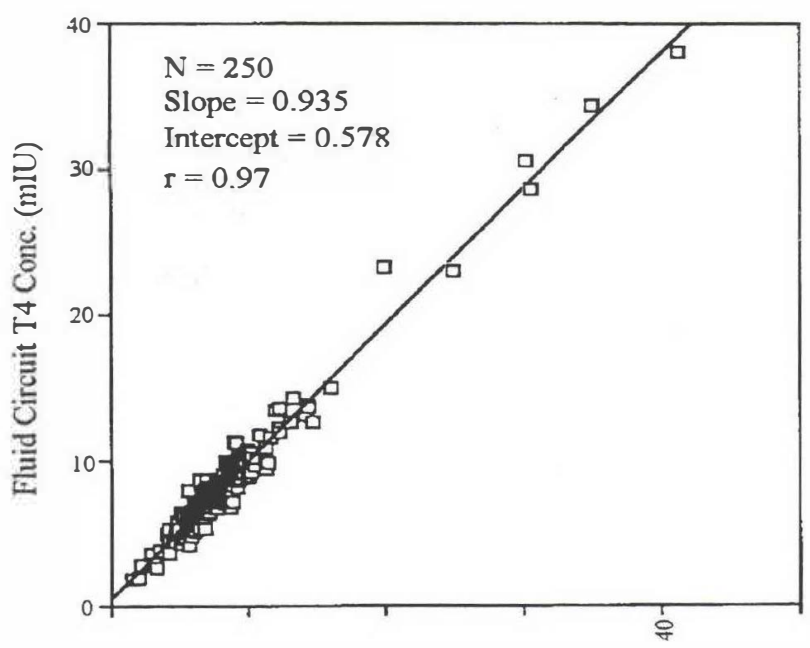

TDx T4 Conc. (mIU)

Fig. 9 Fluid Circuit vs. TDx Comparison

fluid metering/mixing, manipulation, incubation and detection steps for each of the $\mathrm{TD}_{\mathrm{x}}{ }^{\pi}$ assays were evaluated. The critical timing for each of these was assessed, and a sequence of 7 fluid processing loops were developed. Each of these could be performed simultaneously in a pipeline fashion in discrete fluid circuit modules. Four different module types were conceived, as well as a connection structure between modules, and a "pin-out" for the connection structure. This resulted in the design of 7 distinct modules that would; 1) acquire sample, 2) dilute the sample, 3) meter and mix a first reagent, 4) pre-incubate the mixture, 5) meter and mix two additional reagents, 6 ) incubate the final mixture, and 7) present the results to an in-line fluorescence polarimeter.

The fluid circuit that was fabricated is pictured in Fig. 10. Although it could have been designed and fabricated to occupy less space, the circuit is still only $58 \mathrm{~cm} \times 18 \mathrm{~cm} \times 5 \mathrm{~cm}$. There are a total of 254 valves and 24 air/liquid sensors. When operated with a real time multitasking control system the cycle time on the longest fluid processing loop is $\leq 90 \mathrm{sec}$ ( 45 tests/hr. throughput). This fluid circuit system reduces the volume of any single reagent or sample required to $5 \mu \mathrm{L}$ (1/5 of $\mathrm{TD}_{\mathrm{x}}{ }^{\mathrm{n}}$ volumes), requires no plastic disposables, and can continuously perform tests for as long as samples are presented and reagent is available.

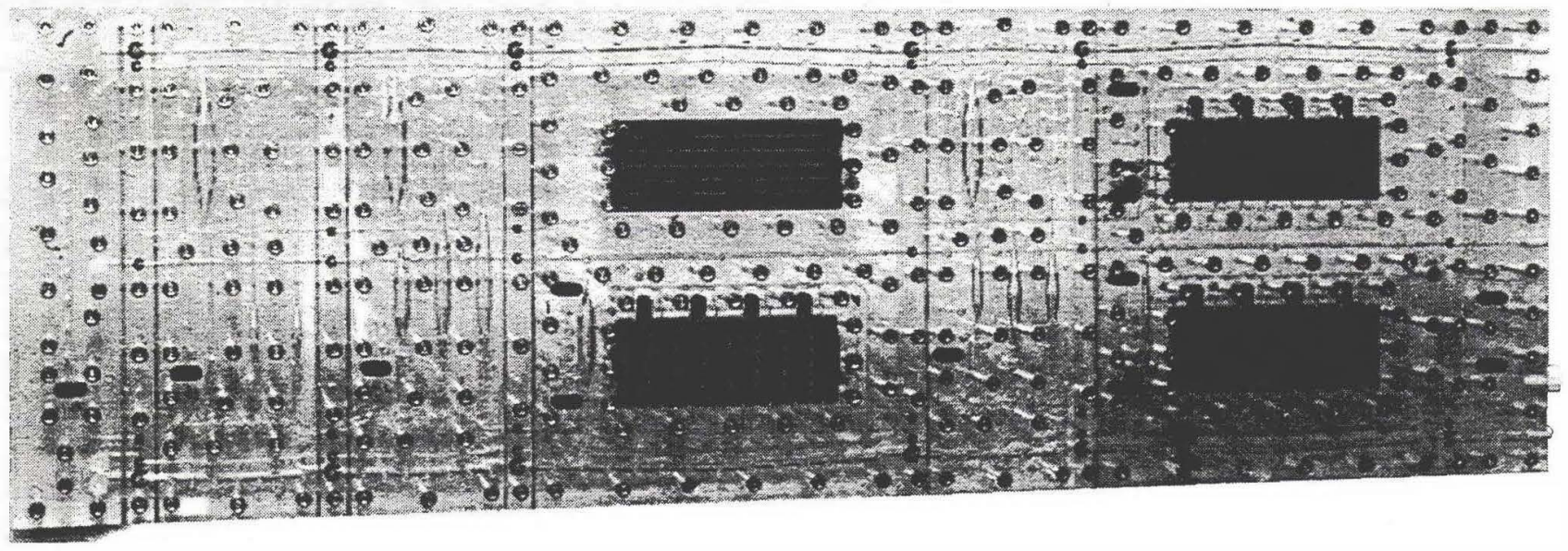

Fig. $10 T D_{x}{ }^{11}$ Equivalent Fluid Circuit System 


\section{RESULTS}

The results of comparing just one of the assays run on this fluid circuit system are illustrated in Fig. 9. This study took $>250$ random clinical patient samples and $\operatorname{ran} \operatorname{TD}_{x}{ }^{\mathrm{TM}} \mathrm{T} 4$ fluorescence polarization immunoassays in triplicate on both the $\mathrm{TD}_{x}{ }^{\mathrm{T}}$ instrument, and the fluid circuit equivalent. These and other results proved that the fluid circuit system performed comparable to the $\mathrm{TD}_{\mathrm{x}}{ }^{\mathrm{Tu}}$ instrument. Similar results were obtained for $\mathrm{THC}$, Total T3, Theophyline and Digoxin assays. Each of these tests stress a diagnostic analyzer in different ways: THC (Cannabis) stresses a fluid handling systems ability to prevent carryover; T3, T4, and Digoxin stress the system's sensitivity and specificity.

Although the fluid circuit system as fabricated and tested did not quite measure up to the $\mathrm{TD}_{\mathrm{x}}$ in throughput, the cause of this was identified as contention between the two final blocks over valves that were shared during certain protocols. If the final incubation module were redesigned to eliminate this resource contention, the resulting fluid circuit system would have a cycle time of $<45 \mathrm{sec}$ and a sustained 80 tests-per-hour throughput.

\section{APPLICATION TO MEMS}

There are two areas that Fluid Circuit Technology can help broaden the impact MEMS can have in fluidic applications.

First, it can act as a model for the design of fluidic MicroElectroMechanical Systems. Using this technology we have been able to quickly research new fluid circuit designs, develop fluid circuit design rules, and understand the underlying principles that affect scaling down fluid control networks. The manufacturing processes described above are quite similar to the processes for fabricating printed circuit boards and even to some of the processes for microfabricating MEMS. The materials are different, the scale is larger, and photolithography is absent, but the creation of three dimensional structure by bonding detail created on two dimensional planes (even the bonding process itself), is so similar that it enables the use of the same schematic capture and board layout CAE design tools used for designing printed circuits. Even the designs of some of the fluid circuit elements are similar. Pneumatically actuated valve designs similar to those we have described here have been microfabricated and remotely controlled [9]. These similarities should enable fluidic MEMS designers to first model their concepts on a macro-scale to visualize the fluid flow and develop an understanding of design issues, before they attempt to shrink their design into an opaque silicon device. Some of the subsystems already described here are ideal candidates for significant further miniaturization. The miniature 16 valve manifold, for instance, while it is already very small, could be integrated and miniaturized to micro-scale if rapid $(5 \mathrm{~ms})$, low power $(125 \mathrm{~mW})$, inexpensive $(<\$ 20)$, and reliable pneumatic valves could be microfabricated.

Second, Fluid Circuit Technology can serve as an interconnect technology. It should be evident that what has been needed to expand the applicability of MEMS into medical uses has been the equivalent of a printed circuit board for fluidic engineers to enable bridging the micro to macro scales and to enable interconnection of complex fluid networks. Since acrylic/silicon bonding is feasible [10], and LIGA substrates can be solvent welded to cast acrylic [11], the packaging of microfabricated devices into fluid circuits should be relatively straight forward.

We believe it should be possible to utilize a fluid circuit as described here to directly mount, and provide fluidic interconnection for, microfluidic MEMS. An alternative method might be to develop the analog of a "chip-carrier" plastic fluid circuit package to which the microfabricated fluidic device could be bonded. The "chip carrier" could serve three purposes. It could provide; macro to micro fluidic interconnect and fluid access ports on one flat gasketed surface, a wire bonding lead frame for electronic connection and, mounting holes and rigidity so that it could be directly bolted to a fluid circuit.

\section{CONCLUSIONS}

The fundamental manufacturing processes, devices, subsystems, and systems that make up Fluid Circuit Technology have been described. Prototype fluid circuit systems have been fabricated to demonstrate the technology's applicability to many of the traditional diagnostic instrument markets, including clinical chemistry, therapeutic drug monitoring, immunochemistry, and hematology. Just as in the $\mathrm{TD}_{\mathrm{x}}{ }^{\mathrm{T}}$ example discussed, in each application it has been evident that this technology is capable of delivering reduction in size, manufacturing costs, consumption of reagent, and the need for disposables, while demonstrating comparable or improved performance and throughput. The precise manipulation of fluid volumes between $1 \mu \mathrm{l}$ and $20 \mathrm{ml}$ to accomplish dilution, metering, mixing, incubation, and detection operations have also been demonstrated.

By drawing parallels between fluid circuit bonding and silicon fusion bonding and between macro and micro pneumatically actuated membrane valves, it may be possible to utilize many of the fluid circuit subsystem designs to build analogous microfabricated fluid handling systems. The direct bonding of microfabricated elements into a fluid circuit system should also be possible, by capitalizing on previous work with bonding acrylic to common MEMS materials. We believe that the use of Fluid Circuit Technology to handle bulk fluid movement in a mixed macro- and micro-scale instrument as well as to provide fluidic access to microfabricated elements is the most feasible and attractive solution currently available and will be just as important to the success of fluidic MEMS as the PC board has been to integrated circuits.

\section{REFERENCES}

1. Schumann, E., MEMS Industry Survey, 1995, SEMI, Mountain View, CA.

2. Berins, M. L., Plastics Engineering Handbook, Society of the Plastics Industry, Van Nostrand Reinhold, New York, NY, 1991, pp. 693-750.

3. Brackett, T., "Method of Bonding Plastics", US Patent $\# 4,875,956$

4. Polycast Technology Corporation, Stamford, CT 06904-0141

5. Flexcon Company Inc., Spencer, MA 01562

6. Webster, M., "Valve with Flexible Sheet Member", US Patent \#4848722, \#4852851, \#4858883

7. The Lee Company, Westbrook, CT, 06498-0424

8. VerLee, D., et.al., "Miniature Liquid/Air Interface Sensor", Proc. of O E M '95, Chicago, IL, SPIE Vol. 2622, 1995.

9. Vieider, C., et. al., "A Pneumatically Actuated Micro Valve with a Silicone Rubber Membrane for Integration with FluidHandling Systems," Digest of Technical Papers The 8th International Conference on Solid-State Sensors and Actuators, Stockholm, Sweden, June 25-29, 1995, vol. 2, pp. 284-285.

10. Brown, Chappell, "Common acrylic is ideal wafer bonder," Electronic Engineering Times, Nov. 14, 1994, Issue 823, p.43.

11. Guckel, H., et. al., "Laterally Driven Electromagnetic Actuators," Tech. Dig. IEEE Solid-State Sensor and Actuator Workshop, Hilton Head Island, SC, Jun 13-16, 1994, pp. 49-52. 